\title{
Correction to: Some characterizations of the tensor product of complete lattices with applications to quantales
}

\author{
Javier Gutiérrez García ${ }^{1}$ • Ulrich Höhle ${ }^{2}$ · Tomasz Kubiak ${ }^{3}$
}

Received: 14 January 2020 / Accepted: 15 January 2020 / Published online: 10 February 2020

(c) Springer Science+Business Media, LLC, part of Springer Nature 2020

\section{Correction to: Semigroup Forum (2019) 99:303-316 https://doi.org/10.1007/s00233-018-9967-9}

For $L$ a complete lattice, let $\operatorname{Up}(L)$ be the complete lattice of all upclosed subsets of $L$ ordered by $\subseteq$ op. An upclosed subset $A$ is prime if $a \vee b \in A$ implies $a \in A$ or $b \in A$. Let $\mathrm{PUp}(L)$ be the complete lattice of all prime members of $U p(L)$.

We apologize for the false statement on page 310 that $\operatorname{Up}(L) \otimes M$ and $\mathrm{PUp}(L) \otimes M$ are always order-isomorphic. Indeed, $M=\mathbf{2}$ is the unit of the tensor product. Thus, if that statement were true, this would mean that $\operatorname{Up}(L)$ and $\operatorname{PUp}(L)$ are order-isomorphic. But this is not always the case as simple counterexamples show. For instance, if $L$ is the four-point diamond, then $\operatorname{PUp}(L)$ has five elements, while $\operatorname{Up}(L)$ has six elements.

That unfortunate statement played a role at the beginning of the proof of Corollary 2. Since this corollary is perfectly correct, it is the purpose of this note to start the proof of Corollary 2 appropriately. Let $f$ be a tensor of $M \otimes L$. By complete distributivity of $M$ and Lemma 3, $f$ can uniquely be identified with the map determined by (see (4.4)):

Communicated by Jimmie D. Lawson.

The original article can be found online at https://doi.org/10.1007/s00233-018-9967-9.

Ulrich Höhle

uhoehle@uni-wuppertal.de

Javier Gutiérrez García

javier.gutierrezgarcia@ehu.eus

Tomasz Kubiak

tkubiak@amu.edu.pl

1 Departamento de Matemáticas, Universidad del País Vasco UPV/EHU, 48080 Bilbao, Spain

2 Fakultät für Mathematik und Naturwissenschaften, Bergische Universität, 42097 Wuppertal, Germany

3 Wydział Matematyki i Informatyki, Uniwersytet im. Adama Mickiewicza, 61-614 Poznan, Poland 


$$
\mathrm{Up}(L) \stackrel{\psi_{f}}{\longrightarrow} M, \quad \psi_{f}(A)=\bigvee f^{-1}(A), \quad A \in \bigcup \mathrm{p}(L) .
$$

Moreover, since $A=\bigcap_{a \notin A} L \backslash \downarrow a$, the following hold:

$$
\psi_{f}(A)=\bigvee\left(\bigcap_{a \notin A} f^{-1}(L \backslash \downarrow a)\right)=\bigwedge_{a \notin A}\left(\bigvee f^{-1}(L \backslash \downarrow a)\right)=\bigwedge_{a \notin A} \psi_{f}(L \backslash \downarrow a),
$$

i.e., $\psi_{f}$ is uniquely determined by its values at all prime upclosed subsets of the form $L \backslash \downarrow a$. Hence $\psi_{f}$ is completely determined by its restriction to $\operatorname{PUp}(L)$. Thus, Lemma 3 implies that every tensor $f \in M \otimes L$ can uniquely be identified with a map PUp $(L) \stackrel{\varphi_{f}}{\longrightarrow} M$ determined by (4.5).

The remaining part of the original proof of Corollary 2 remains unchanged.

None of the other results of the paper are affected.

Publisher's Note Springer Nature remains neutral with regard to jurisdictional claims in published maps and institutional affiliations. 